\title{
Complex Congenital Heart Disease in an Infant - A Case Report and Review
}

\author{
Srinivas Naik ${ }^{1}$, Sourya Acharya ${ }^{2}$, Gajendra Agrawal $^{3}$, Chetan Rathi' ${ }^{4}$, Sunil Kumar ${ }^{5}$ \\ 1,2,5 Department of Medicine, Datta Meghe Institute of Medical Sciences (Deemed to Be University), \\ Sawangi, Wardha Maharashtra, India. ${ }^{3,4}$ Department of Cardiology, Datta Meghe Institute of Medical \\ Sciences (Deemed to Be University), Sawangi, Wardha Maharashtra, India.
}

\section{INTRODUCTION}

Congenital heart disease (CHD) often poses a great diagnostic challenge for physicians. Despite antenatal diagnostic tests advancing to a great level, accurate diagnosis and treatment of congenital heart diseases is mandatory. These diseases range from mild to severe life-threatening scenarios sometimes having vague presentations making diagnosis even more difficult. Early diagnosis and treatment are usually lifesaving. ${ }^{1}$

Congenital heart diseases can often be classified as cyanotic and acyanotic based on clinical presentation. After birth, fetal structures like foramen ovale, ductus venosus and ductus arteriosus are no longer required for survival and they begin to close. ${ }^{2}$ Persistence of such structures after birth is a sign of congenital heart diseases. High mortality contributing diseases which require prompt intervention include hypoplastic left heart syndrome (HLHS), coarctation of aorta (COA), interrupted aortic arch (IAA), transposition of the great arteries (TGA), total anomalous pulmonary venous return (TAPVR), critical aortic stenosis (AS) pulmonary atresia (PA) and tricuspid atresia (TA). ${ }^{3}$ They contribute to significant mortality amongst neonatal age groups.

Recognition of congenital heart diseases based on clinical fractures like cyanosis, tachycardia, tachypnoea, irritability, refusal to feed stabilisation and prompt referral to tertiary cardiac centre are critical to improve outcomes in neonates with CHDs, seizures, murmur etc is diagnostically challenging but lifesaving. Life-threatening CHDs may perhaps present with cyanosis, respiratory distress, shock or collapse; all of these are also frequent clinical presentation of various respiratory problems or sepsis in newborn. Early diagnosis and prompt treatment are the only life saving measures. ${ }^{4}$

\section{PRESENTATION OF CASE}

A 10-month-old infant suffering from a case of large ostium primum atrial septal defect (ASD) and ventricular septal defect came to the casualty with complaints of fever, refusal to feed and vomiting for 2 days. Patient was apparently alright 2 days ago and then started having fever which according to the mother was sudden in onset. Fever was not associated with chills and rigor. Mother stated that patient also had refusal to feed and had 3 episodes of vomiting. On general examination, patient was febrile with temp of $103^{\circ} \mathrm{F}$ with a pulse of 146 per minute, regular in rhythm, respiratory rate of 40 per minute and blood pressure of 90/70 $\mathrm{mm}$ hg in right arm supine position. Cyanosis and clubbing were absent. His laboratory investigations are mentioned in table number one. Patient was treated with antibiotics and other supportive measures were given. Chest $\mathrm{x}$ ray showed cardiomegaly.
Corresponding Author: Dr. Sourya Acharya, Professor and Head, Department of Medicine, Datta Meghe Institute of Medical Sciences (Deemed to Be University), Sawangi, Wardha Maharashtra, India. E-mail: souryaacharya74@gmail.com

DOI: $10.14260 /$ jemds/2021/710

How to Cite This Article:

Naik S, Acharya S, Agrawal G, et al. Complex congenital heart disease in an infant - a case report and review. J Evolution Med Dent Sci 2021;10(39):3505-3507, DOI: 10.14260/jemds/2021/710

Submission 13-05-2021,

Peer Review 04-08-2021,

Acceptance 30-08-2021,

Published 27-09-2021.

Copyright @ 2021 Srinivas Naik et al. This is an open access article distributed under Creative Commons Attribution License [Attribution 4.0 International (CC BY 4.0)] 
Transthoracic echocardiography revealed large ostium primum atrial septal defect $(20 \mathrm{~mm})$ with severe $M R$, moderate TR with severe PAH and was planned for surgery (ASD closure + VSD closure + mitral valve repair + tricuspid valve repair) under general anaesthesia. - Chest $\mathrm{X}$ ray PA view showed cardiomegaly and pulmonary plethora.

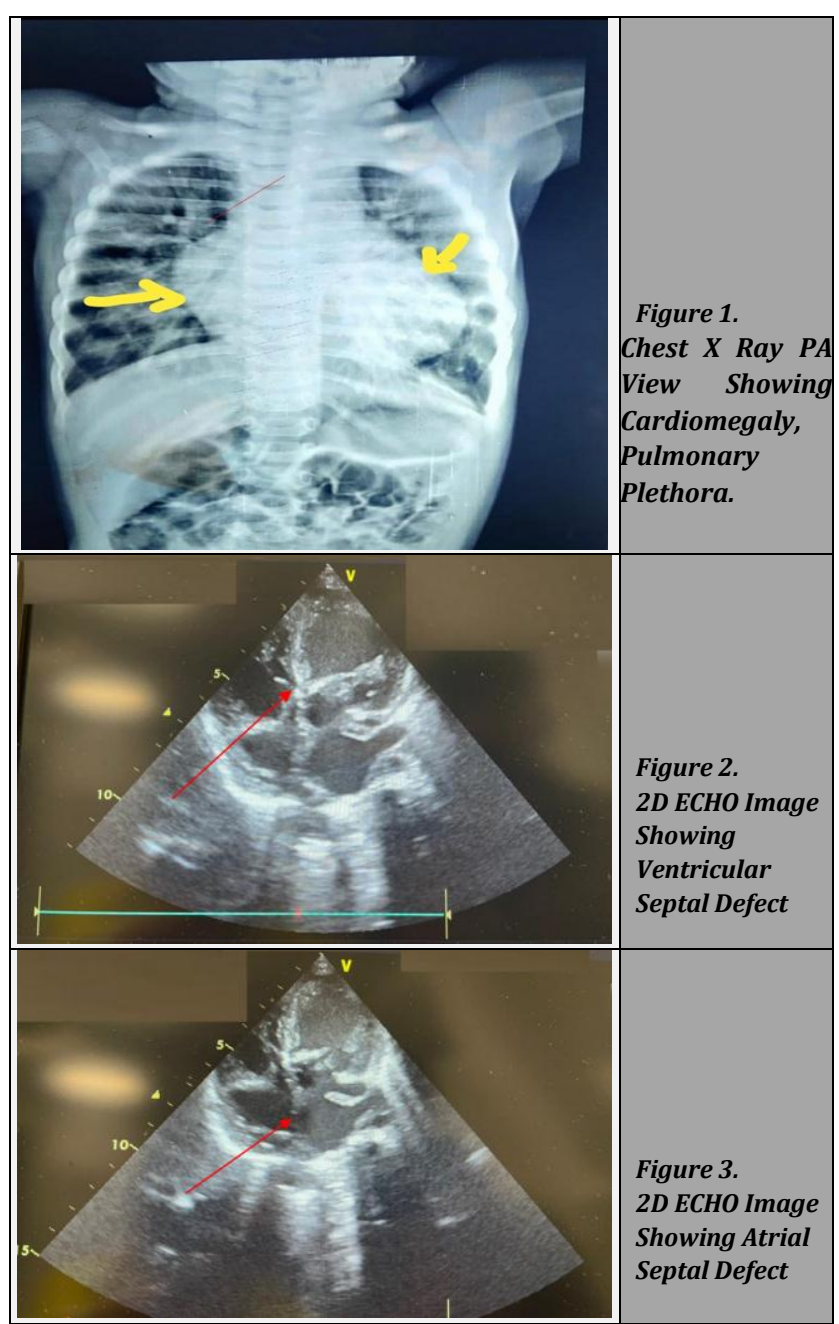

DISCUSSION

It has been established that atrial septal abnormalities can worsen a baby's respiratory state and necessitate more oxygen and/or ventilatory support. Surgical and catheter closure of such abnormalities improves breathing quickly, allowing for early extubation and oxygen weaning. The causes of increased pulmonary blood flow (ASD, VSD) almost certainly contributed to the severity of the condition. Even though there is a higher prevalence of congenital coronary heart defects which has been reported in patients with chromosomal abnormalities, such as those with chromosome eight defects, it is unclear whether the underlying medical image in our patient became intrinsically linked to the excessive material in chromosome eight. ${ }^{5}$

The natural history of fundamental problems, the existence of excessive pulmonary high blood pressure, including the morbidity of the to be had surgical and interventional techniques were all taken into consideration during the decision-making process.
Although percutaneous closure of congenital abnormalities has been performed in neonates and even preterms, the risk of complications, such as arterial occlusion and tool protrusion causing both left pulmonary artery stenosis and coarctation, is lower at this age group than at higher ages. Also, from a technical standpoint, the system isn't always as smooth as it should be, and there are some gadgets that aren't up to par.

Because ASDs rarely induce any significant symptomatology in small children, the experience is limited with percutaneous closure of those defects in this age group, particularly in small neonates. ${ }^{6}$ Patients who seek to close their ASD at a younger age and go through this type of treatment are more likely to have comorbid conditions such as genetic disorders and non-cardiac abnormalities, as well as preterm. Defects without anterosuperior rim, may also provide demanding situations with respect to positioning of the tool in area, in which greater manipulation of sheaths from the femoral vein and/or software of much fewer fashionable strategies are required. Based on the current reviews of a success preliminary palliation of neonates with hypoplastic coronary heart syndrome and greater importantly, periventricular closure of muscular VSDs the hybrid technique has emerged as an appealing opportunity to cope with congenital defects on the equal time with inside the case offered here. ${ }^{7}$

Although periatrial ASD closure has been executed in advance in a baby with a related muscular VSD, it is profitable in discussing few technical factors of the system. We experience that the most important thing that trouble for a success implantation changed into the site for puncturing the proper atrial wall below proper atrial appendage. In this way, the left atrial disk should approach the interatrial septum at a parallel perspective, heading off prolapse through the poor anterosuperior rim. For a couple of reasons now we no longer determined to balloon length the defect. Primarily, the profile and the lengths of the available sizing balloons might have built the sizing system, through a periatrial technique, a cumbersome or maybe not possible one, in this kind of small infant.6,7 Secondarily, the ASD closure has been executed without stretching the defect through the use of gadgets which might be $10-30 \%$ larger than it. Therefore, we arbitrarily selected a device that changed into approximately $10-15 \%$ large than the defect, deliberating that the left disk might in shape with inside the left atrium. Also, the surest perspective of attack closer to the interatrial septum helped to area an 'undersized' tool correctly. With respect to left disk manipulation, we felt that the same old TEE probe, considering its length and the near region to the oesophagus and the posterior left atrial wall, exerted few compression impact at the left atrium and will have impaired left disk deployment. This won't be a problem in small babies although the intra cardiac probe (AcuNav) is used for TEEs. Also, due to the fact that there has been no longer sufficient room with inside the proper atrium, the method of deployment of the proper atrial disk needed to be barely modified. At a slow pace, retracting of sheath and pushing at the delivery cable, proper disk changed into reconfigured near the septum and at a great distance from the access site in the proper atrial wall. Ultimately, the TEE tracking with optimal imaging of all of the steps of the system changed into essential for a success wire and sheath manipulations and adequate implantation. 


\section{CONCLUSIONS}

Finally, the case presented here shows how the so-called 'hybrid method' can be used in successfully managing a highrisk new-born with severe pulmonary hypertension and several congenital heart abnormalities along with a left to right shunt without the need for cardiopulmonary bypass. The field of hybrid methods to complex congenital heart disease care is rapidly expanding. This eliminates the need for cardiopulmonary bypass surgery. The field of hybrid methods to the care of complicated congenital heart disease is rapidly expanding. This is especially important in cases where certain problems cannot be treated easily and safely in the surgical suite or catheterization laboratory due to patient or anatomical factors. As a result, the surgeon's competence is required.

Financial or other competing interests: None.

Disclosure forms provided by the authors are available with the full text of this article at jemds.com.

\section{REFERENCES}

[1] Taussig HB, Blalock A. The tetralogy of Fallot: diagnosis and indications for operation: the surgical treatment of the tetralogy of Fallot. Surgery 1947;21(1):145.

[2] Lewis FJ, Taufic M. Closure of atrial septal defects with the aid of hypothermia; experimental accomplishments and the report of one successful case. Surgery 1953;33(1):529.

[3] Lillehei CW, Cohen M, Warden HE, et al. The direct - vision intracardiac correction of congenital anomalies by controlled cross circulation; results in thirty - two patients with ventricular septal defects, tetralogy of Fallot and atrioventricularis communis defects. Surgery 1955;38(1):11-29.

[4] Gibbon JH Jr. Application of a mechanical heart and lung apparatus to cardiac surgery. Minn Med 1954;37(3):17185, passim.

[5] Kasturi L, Kulkarni AV, Anin A, et al. Congenital heart disease: clinical spectrum. Indian Pediatr 1999;36(9):953.

[6] Khalil A, Aggarwal R, Thirupuram S, et al. Incidence of congenital heart disease among hospital live births in India. Indian Pediatr 1994;31(5):519-27.

[7] Michel-Behnke I, Ewert P, Koch A, et al. Device closure of ventricular septal defects by hybrid procedures: a multicenter retrospective study. Catheter Cardiovasc Interv 2011;77(2):242-51. 